\title{
Article \\ Delineating the Tnt1 Insertion Landscape of the Model Legume Medicago truncatula cv. R108 at the Hi-C Resolution Using a Chromosome-Length Genome Assembly
}

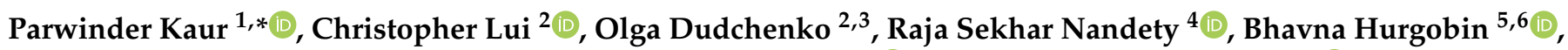
Melanie Pham ${ }^{2,3}$, Erez Lieberman Aiden ${ }^{1,2,3,7,8}$, Jiangqi Wen ${ }^{4}(\mathbb{D})$ and Kirankumar S Mysore $4, *(\mathbb{B})$

1 UWA School of Agriculture and Environment, The University of Western Australia, Crawley, WA 6009, Australia; erez.Lieberman@bcm.edu

2 The Center for Genome Architecture, Department of Molecular and Human Genetics, Baylor College of Medicine, Houston, TX 77030, USA; christopher.g.lui@gmail.com (C.L.); olga.Dudchenko@bcm.edu (O.D.); melanie_pham@ymail.com (M.P.)

3 Center for Theoretical and Biological Physics, Departments of Computer Science and Computational and Applied Mathematics, Rice University, Houston, TX 77030, USA

4 Noble Research Institute, LLC., Ardmore, OK 73401, USA; rsnandety@noble.org (R.S.N.);

check for

updates

Citation: Kaur, P.; Lui, C. Dudchenko, O.; Nandety, R.S.; Hurgobin, B.; Pham, M.; Lieberman Aiden, E.; Wen, J.; Mysore, K.S Delineating the Tnt1 Insertion Landscape of the Model Legume Medicago truncatula cv. R108 at the Hi-C Resolution Using a

Chromosome-Length Genome Assembly. Int. J. Mol. Sci. 2021, 22, 4326. https://doi.org/10.3390/ ijms22094326

Academic Editors: Fatemeh Maghuly, Eva M. Sehr, Rachit Saxena and David J. Konkin

Received: 17 March 2021

Accepted: 15 April 2021

Published: 21 April 2021

Publisher's Note: MDPI stays neutral with regard to jurisdictional claims in published maps and institutional affiliations.

Copyright: (c) 2021 by the authors. Licensee MDPI, Basel, Switzerland. This article is an open access article distributed under the terms and conditions of the Creative Commons Attribution (CC BY) license (https:// creativecommons.org/licenses/by/ $4.0 /)$. jwen@noble.org (J.W.)

5 La Trobe Institute for Agriculture and Food, Department of Animal, Plant and Soil Sciences, School of Life Sciences, AgriBio Building, La Trobe University, Bundoora, VIC 3086, Australia; bhavna.hurgobin@hotmail.com

6 Australian Research Council Research Hub for Medicinal Agriculture, AgriBio Building, La Trobe University, Bundoora, VIC 3086, Australia

7 Broad Institute of MIT and Harvard, Cambridge, MA 02139, USA

8 Shanghai Institute for Advanced Immunochemical Studies, ShanghaiTech, Pudong 201210, China

* Correspondence: parwinder.kaur@uwa.edu.au (P.K.); ksmysore@noble.org (K.SM.); Tel.: +61-8-6488-7120 (P.K.); +1-580-224-6740 (K.SM.)

Abstract: Legumes are of great interest for sustainable agricultural production as they fix atmospheric nitrogen to improve the soil. Medicago truncatula is a well-established model legume, and extensive studies in fundamental molecular, physiological, and developmental biology have been undertaken to translate into trait improvements in economically important legume crops worldwide. However, M. truncatula reference genome was generated in the accession Jemalong A17, which is highly recalcitrant to transformation. M. truncatula R108 is more attractive for genetic studies due to its high transformation efficiency and Tnt1-insertion population resource for functional genomics. The need to perform accurate synteny analysis and comprehensive genome-scale comparisons necessitates a chromosome-length genome assembly for M. truncatula cv. R108. Here, we performed in situ Hi-C $(48 \times)$ to anchor, order, orient scaffolds, and correct misjoins of contigs in a previously published genome assembly (R108 v1.0), resulting in an improved genome assembly containing eight chromosome-length scaffolds that span $97.62 \%$ of the sequenced bases in the input assembly. The long-range physical information data generated using Hi-C allowed us to obtain a chromosomelength ordering of the genome assembly, better validate previous draft misjoins, and provide further insights accurately predicting synteny between A17 and R108 regions corresponding to the known chromosome 4/8 translocation. Furthermore, mapping the Tnt1 insertion landscape on this reference assembly presents an important resource for $M$. truncatula functional genomics by supporting efficient mutant gene identification in Tnt1 insertion lines. Our data provide a much-needed foundational resource that supports functional and molecular research into the Leguminosae for sustainable agriculture and feeding the future.

Keywords: Leguminosae; Medicago truncatula cv. R108; HiC; chromosome-length genome assembly; Tnt1 insertion landscape 


\section{Introduction}

Sustainable agricultural production involves growing food with low fertilizer input without damaging the underlying soil [1]. Legumes are of great interest for sustainable agriculture because they produce nitrogen via symbiotic nitrogen fixation, improving soil health [2,3]. Most legumes, however, have large/complex genomes and are outcrossing species, making genetic studies difficult. Medicago truncatula was chosen as a model legume due to its small genome [4], diploidy, autogamy, and short life cycle. In the past two decades, extensive studies have been undertaken in plant-bacterial symbioses and fundamental molecular, physiological, and developmental biology of M. truncatula to translate and improve traits in economically important legume crops [5-9]. The release of the $M$. truncatula accession Jemalong A17 reference genome sequence and generation of the Tnt1-based insertion mutant population for accession R108 have greatly accelerated functional genomics studies in M. truncatula [10-12]. The M. truncatula reference genome was generated in A17, which is highly recalcitrant to transformation, whereas the Tnt1 mutant population was generated in R108, with a much higher transformation efficiency. Phylogenetically, R108 is one of the most distant M. truncatula accessions from A17 [13]. R108 is more attractive for genetic studies due to its high transformation efficiency [10]. Recently, R108 has become popular in legume research communities with its near-saturated Tnt1-insertion population, which is widely used in most areas of legume functional genomic analysis $[10,12]$. The $T n t 1$ insertion population comprises 21,700 regenerated lines, encompassing more than a half-million randomly distributed Tnt1 insertions [12]. Due to the lack of high-quality pseudomolecules (chromosomes) in R108, all Tnt1 insertions are mapped to the A17 genome. However, compared to R108 and other M. truncatula genotypes, A17 has a large $(\sim 30 \mathrm{Mb})$ reciprocal translocation between chromosomes 4 and 8 [4], resulting in inaccurate synteny analysis between $M$. truncatula and other legume genomes and aberrant recombination in genetic crosses, including crosses between A17 and R108 [14]. In addition, evolutionary whole-genome duplications $[13,15]$ and frequent local duplications make genome assembly difficult. Therefore, having two high-quality references in M. truncatula will allow us to perform more accurate synteny analysis and comprehensive genome-scale comparisons, and calls for the M. truncatula cv. R108 genome sequence.

Three years ago, the first draft assembly of M. truncatula cv. R108 was constructed using a combination of PacBio, Dovetail, and BioNano technologies, as described by Moll et al. (2017). Recently, we and others significantly improved draft genomes using data derived from in situ Hi-C [16-19]. As Hi-C can estimate the relative proximity of loci in the nucleus, Hi-C contact maps can be used to correct misjoins, anchor, order, and orient contigs and scaffolds. This process improves contig accuracy and typically yields chromosomelength scaffolds. To broaden the range of genetic resources available for the model legume M. truncatula, we used Hi-C to improve the R108 v1.0 draft assembly, producing a genome assembly for M. truncatula cv. R108 with chromosome-length scaffolds. Approximately 387,000 flanking sequence tags (FSTs), identified from approximately 21,000 Tnt1 insertion lines of M. truncatula cv. R108, were mapped onto pseudo-chromosomes of R108.

\section{Results}

\subsection{Assembly of M. truncatula Accession R108 with Chromosome-Length Scaffolds}

The first draft assembly of $M$. truncatula $\mathrm{cv}$. R108 was constructed using a combination of PacBio, Dovetail, and BioNano technologies [20]. The resulting assembly (R108 v1.0) comprised $402 \mathrm{Mb}$ of sequence (contig N50 length: $5.93 \mathrm{Mb}$ ) partitioned among 909 scaffolds. Here, we generated in situ Hi-C data $[16,18]$ from $M$. truncatula cv. R108 leaves to improve its initial draft assembly $[19,21]$. Scaffolds/contigs shorter than $1 \mathrm{~Kb}$ were not anchored from the R108 v1.0 assembly; the remaining scaffolds were anchored, ordered, oriented, and corrected for misjoins using the Hi-C data. After manual refinement using Juicebox Assembly Tools [21], as shown in Figure 1, the resulting assembly, named MedtrR108_hic, was represented by 801 scaffolds, of which eight were chromosome-length scaffolds (N50 length of $51.86 \mathrm{Mb}$ ), ranging from 37.80 to $55.90 \mathrm{Mb}$. The chromosome-length 
scaffolds spanned $97.62 \%$ of the sequenced bases in the entire assembly. The remaining 793 scaffolds (N50 length of $18.96 \mathrm{~Kb}$ ) constituted the remaining $2.38 \%$ of the total assembly. The circular snail plots describing the assembly statistics of MedtrR108_hic and R108 v1.0 are shown in Figure 2a,b. These results are further summarized in Table 1. Additional assembly statistics can be found in Tables S1-S5.

Assessment of the Hi-C genome assembly quality was performed via CEGMA analysis [22] to identify the presence of core eukaryotic genes (CEGs). From the 248 CEGs analyzed, $228(91.94 \%)$ complete genes and $245(98.79 \%)$ partial genes were identified in the Hi-C assembly (Table S6). In contrast, only $223(89.92 \%)$ and $242(97.52 \%)$ complete and partial CEGs were found in the R108 v1.0 assembly (Table S6).

Table 1. Assembly statistics for the MedtrR108_hic genome assembly. Note that scaffolds smaller than $1 \mathrm{Kbp}$ are excluded from the analysis.

\begin{tabular}{cc}
\hline Statistics & MedtrR108_hic \\
\hline Draft scaffolds & $399,348,955$ \\
Base pairs & 1005 \\
Number of contigs & $5,925,378$ \\
Contig N50 & 909 \\
Number of scaffolds & $12,848,239$ \\
Scaffold N50 & \\
Chromosome-length scaffolds & $390,045,474$ \\
Base pairs & 209 \\
Number of contigs & $6,045,855$ \\
Contig N50 & 8 \\
Number of scaffolds & $51,860,634$ \\
Scaffold N50 & \\
Small scaffolds & $5,840,890$ \\
Base pairs & 248 \\
Number of contigs & 24,000 \\
Contig N50 & 236 \\
Number of scaffolds & 24,736 \\
Scaffold N50 & \\
Tiny scaffolds & $3,462,591$ \\
Base pairs & 558 \\
Number of contigs & 9246 \\
Contig N50 & 557 \\
Number of scaffolds & 9246 \\
Scaffold N50 & \\
\hline & \\
\hline & \\
\hline & \\
\hline & \\
\hline &
\end{tabular}




\section{$\odot$}

R108 v. 1.0

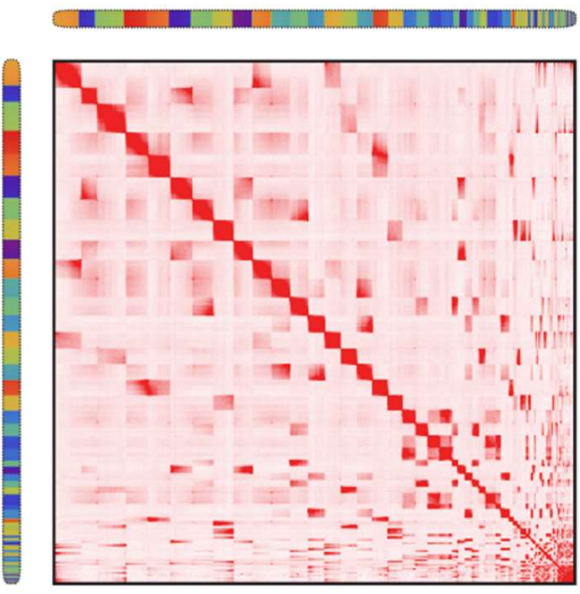

Draft assembly: Moll et al. BMC Genomics (Aug 2017) (PacBio DNA-Seq + Bionano + Dovetail)
R108 v. HiC

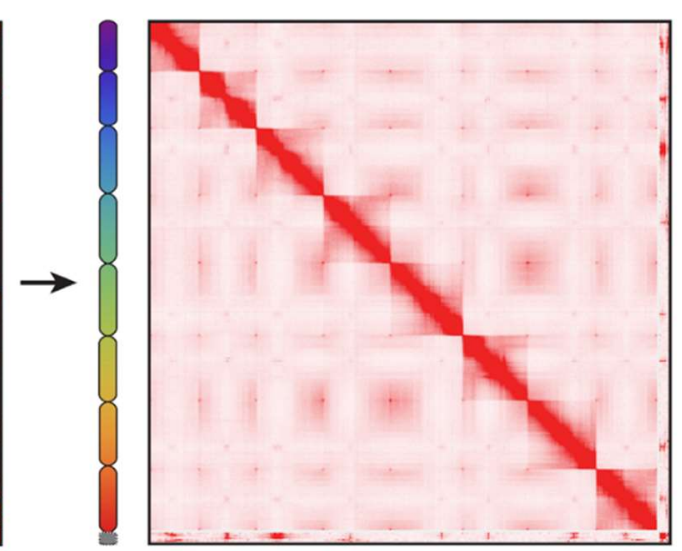

Chromosome-length assembly: MedtrR108_hic

Figure 1. Hi-C map of the draft and chromosome-length assemblies of Medicago truncatula cv. R108 genome. Contact matrices were generated by aligning the same Hi-C data set to the R108 v1.0 draft genome (left) and MedtrR108_hic genome assembly generated using Hi-C (right). Pixel intensity in the matrix indicates how often a pair of loci co-locate in the nucleus. Correspondence between loci in the draft and final assemblies is illustrated using chromograms. The chromosome-length assembly scaffolds in Med-trR108_hic are assigned a linear color gradient. hic are assigned a linear color gradient; the same colors are then used for the corresponding loci in the R108v1.0 (left). The draft scaffolds are ordered by sequence name. Gridlines highlight the boundaries of eight chromosome-length scaffolds in MedtrR108_hic (right). Scaffolds smaller than $10 \mathrm{~kb}$ in R108v1.0 are not included.
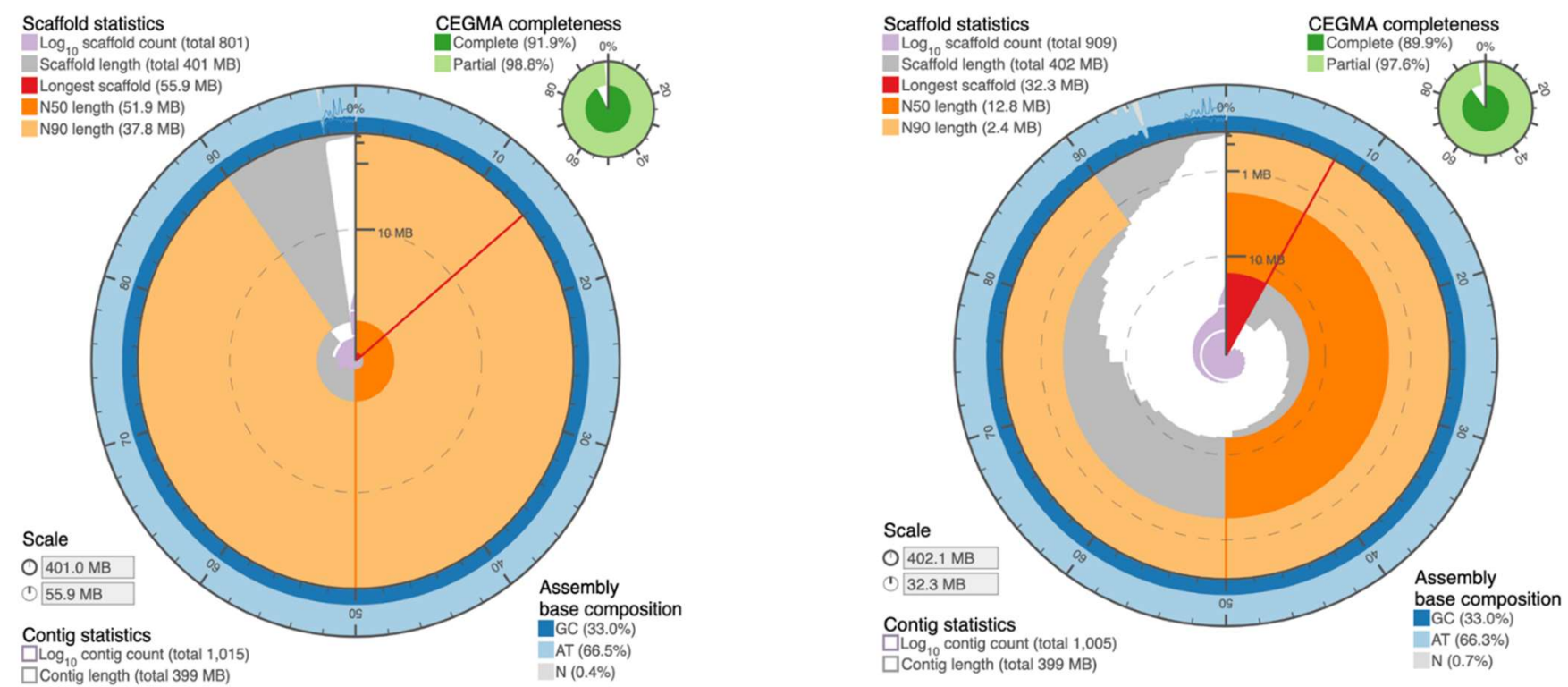

Figure 2. Snail plots describing the assembly statistics of the (A) MedtrR108_hic assembly and (B) R108 v1.0 assembly. Note the larger values for the longest scaffolds, N50 and N90, for MedtrR108_hic than R108 v1.0. The plots were generated using https:/ / github.com/rjchallis/assembly-stats, accessed on 17 March 2021. 


\subsection{Genome Annotation and Functional Characterization}

Reannotation of the MedtrR108_hic genome assembly predicted 39,027 high-confidence, protein-coding genes, which is lower than the 55,706 and 44,623 protein-coding genes annotated in the R108 v1.0 (GenBank accession no. GCA_002024945.1) and A17 Mt5.0 assemblies (GenBank accession no. GCA_003473485.2), respectively [14,20]. However, assessment of gene space completeness via a Benchmarking Universal Single-Copy Orthologs (BUSCO) analysis [23] showed that the MedtrR108_hic assembly harbored a higher percentage of complete BUSCOs (96.73\%) than the R108 v1 assembly (91.94\%) among the 2326 BUSCO groups searched (Table S7). The percentages of fragmented and missing BUSCOs were also less in the MedtrR108_hic assembly than the R108 v1.0 assembly. Further, the number of complete BUSCOs (single copy and duplicated) were more comparable between MedtrR108_hic and A17 Mt5.0 than between R108 v1 and A17 Mt5.0.

Of the 39,027 genes, 36,994 (94.79\%) had at least one hit against either A17 Mt5.0 proteins [14], TAIR10 (https: / /www.arabidopsis.org/, accessed on 17 March 2021), Phytozome [24] v13, Swissprot [25], RefSeq [26] or TrEMBL [25] databases, as revealed by the BLAST searches (Table S8). Phytozome v13 and TrEMBL were the most informative databases, assigning functional annotations to $93.53 \%$ and $94.20 \%$ of the predicted genes, respectively. The R108 v1.0 genes had lower percentages of BLAST hits overall ( $84.72 \%)$, further confirming the relatively more complete annotation of the MedtrR108_hic assembly compared to the R108 v1.0 assembly (Table S9).

The MedtrR108_hic protein-coding genes were also mined for protein domains and annotated with gene ontology (GO) terms. A total of 29,504 (75.60\%) and 19,086 (48.90\%) genes had at least one protein domain and GO term assigned to them, respectively (Table S10). All publicly available RNA-Seq accessions used for annotation are presented as supplementary information (Table S11).

\subsection{Synteny Analysis and Chromosomal Translocation}

A high degree of collinearity was observed between A17 Mt5.0 and MedtrR108_hic (Figure $3 \mathrm{a}, \mathrm{b}$ ). However, as reported previously [14,27], translocation between chromosomes 4 and 8 of the A17 genome was visible. More precisely, a $12 \mathrm{Mb}$ syntenic region was observed between A17 Mt5.0 chromosome 4 (46.93-64.75 Mb) and MedtrR108_hic chromosome 8 (32.86-50.23 Mb). Further, a $17 \mathrm{Mb}$ syntenic region between A17 Mt5.0 chromosome 8 (37.03-49.69 Mb) and MedtrR108_hic chromosome 4 (41.12-35.19 Mb) was visible. While the $12 \mathrm{Mb}$ syntenic region was also reported in A17 Mt5.0 versus R108 v1.0 [14], the $17 \mathrm{Mb}$ syntenic region observed in the current study is significantly larger than the $4 \mathrm{Mb}$ syntenic region reported between R108 v1.0 chromosome 8 and A17 Mt5.0 chromosome 4 [14]. Furthermore, Pecrix et al. identified three additional translocations in A17 Mt5.0R108 v1.0, but these were absent from the A17 Mt5.0 versus MedtrR108_hic comparison. As previously reported [14], inversion in the first $8.7 \mathrm{Mb}$ region of chromosome 1 of A17 Mt5.0 was also clearly visible [14]. 


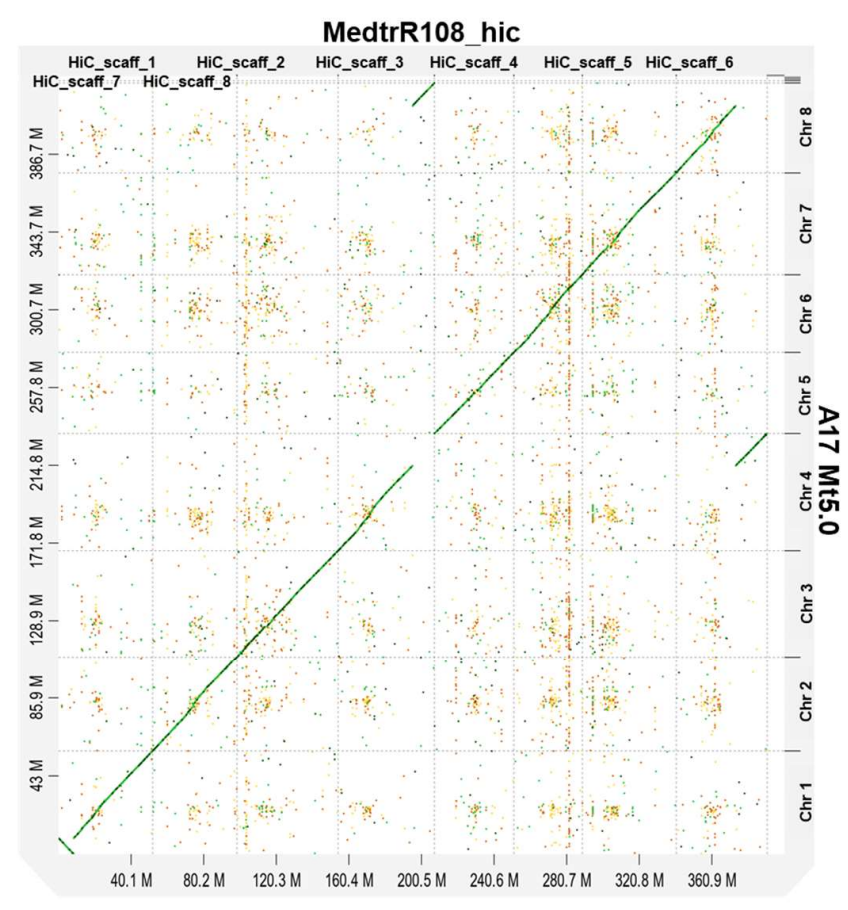

A

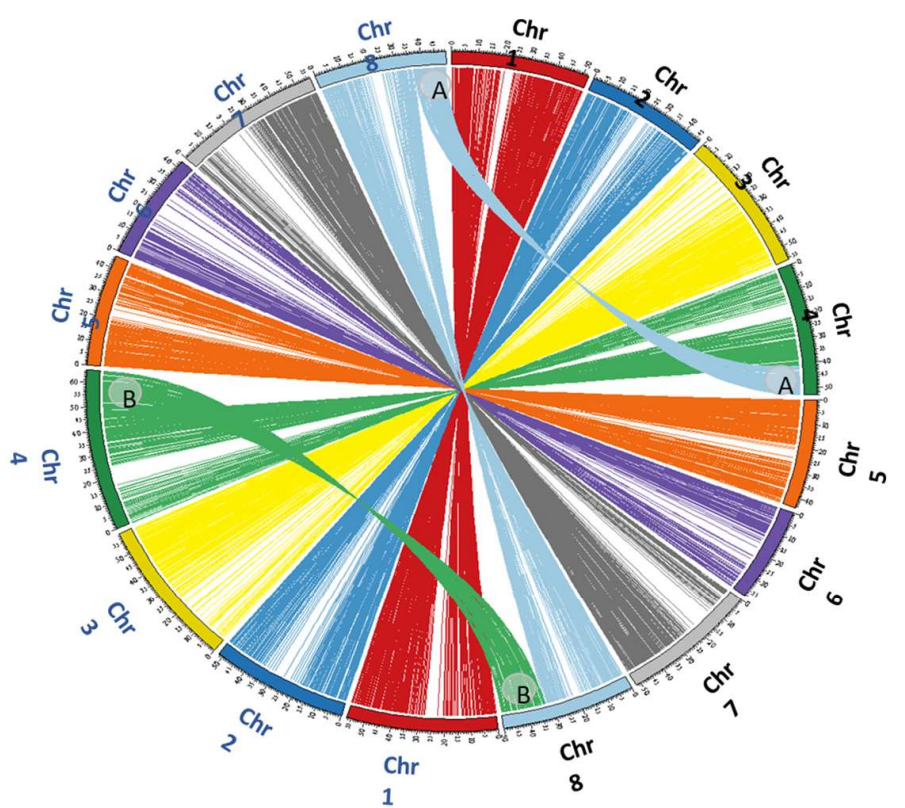

B

Figure 3. Assembly using Hi-C improves comparative analysis. (A) Whole-genome alignments of MedtrR108_hic versus A17 Mt5.0 highlight the peculiarity of the A17 genotype better than those between R108 v1.0 and A17 Mt5.0 [14]. (B) Circos plot depicts the genome structure of the syntenic relationship between MedtrR108_hic (chromosome names on right in black) and A17 Mt5.0 (chromosome names on left in blue) via syntenic links. The translocated regions on chromosomes 4 and 8 are highlighted: A denotes a $12 \mathrm{Mb}$ syntenic region between MedtrR108_hic chromosome 4 (41.1-53.2 Mb) and A17 Mt5.0 chromosome 8 (37-49.7 Mb), and B denotes a $17 \mathrm{Mb}$ syntenic region between MedtrR108_hic chromosome 8 (32.9-50.2 Mb) and A17 Mt5.0 chromosome 4 (46.9-64.7 Mb). The syntenic links represent syntenic blocks that are at least $50 \mathrm{Kbp}$ long, and chromosome sizes are shown in $\mathrm{Mb}$. Only the largest scaffolds/chromosomes determined syntenic relationships.

Overall, the A17 Mt5.0 versus MedtrR108_hic syntenic genes could be arranged in a smaller number of larger blocks than the A17 Mt5.0 versus R108 v1.0 syntenic genes. A total of 25,548 syntenic genes were identified between A17 Mt5.0 and MedtrR108_hic (Table S12), which could be arranged in 59 collinear blocks. The largest block (no. 54) contained 2574 genes, while the smallest block (no. 49) contained four genes (Table S13). In contrast, 26,348 syntenic genes were identified between A17 Mt5.0 and R108 v1.0 (Table S14), which could be arranged into 121 collinear blocks. The largest block (no. 66) contained 1535 genes, while the smallest block (no. 73) contained four genes (Tables S12-S14).

Of the 25,548 syntenic genes identified in A17 Mt5.0 versus MedtrR108_hic, 2676 $(10.47 \%)$ genes were found in the translocated regions (Table S14); of which, 1143 were found in the $12 \mathrm{Mb}$ region, with the remaining 1533 genes in the $17 \mathrm{Mb}$ region (Table S14). Of the 26,348 syntenic genes identified between A17 Mt5.0 and R108 v1.0, 1590 (6.03\%) genes were found in the translocated regions; most of which (1159) were found in the $12 \mathrm{Mb}$ region, while the remaining 431 genes were in the $4 \mathrm{Mb}$ region (Table S14). The GO terms commonly enriched in the translocated regions between A17 Mt5.0 and MedtrR108_hic (Table S15) and A17 Mt5.0 and R108 v1.0 (Table S16) comprised the following stressresponse related-terms: response to water deprivation, plant-type hypersensitive response, response to ethylene, response to abscisic acid, and response to jasmonic acid (Table S17).

\subsection{Mapping Tnt1 Insertion Sites in the M. truncatula R108 Hi-C Genome Assembly}

From the 21,741 Tnt1 insertion lines generated in M. truncatula cv. R108, 392,396 FSTs were recovered using TAIL-PCR and Sanger or Illumina sequencing [12]. The average sequence length of these FSTs is $363 \mathrm{bp}$. To identify the signature sequence in FSTs, we 
processed all FSTs and obtained 221,275 high-confidence FST sequences. The remaining 171,121 FSTs that lacked signature sequence likely resulted from AD primer end sequencing. Of the 221,275 FSTs, 202,788 (92\%) were successfully mapped to the M. truncatula R108 reference genome MedtrR108_hic with an identity greater than 90\% (Table 2). A total of 201,427 Tnt1 insertions were mapped to eight chromosomes, with an average of 25,178 insertions per chromosome (Table 2). The most Tnt1 insertions $(27,902 ; 12.6 \%)$ were mapped onto chromosome 1 and the least $(16,433 ; 7.4 \%$ ) were mapped onto chromosome 6 (Table 2). It is reasonable to observe low numbers on chromosome 6 as it is the smallest of the eight chromosomes. In addition, 1361 Tnt1 insertions were mapped onto the unanchored scaffolds. The mapping of Tnt1 insertions across all R108 chromosomes confirms previous results that showed random Tnt1 insertions based on the M. truncatula A17 genome [12]. All Tnt 1 insertions were mapped to chromosomes in the Hi-C assembly of the R108 genome based on physical chromosome location through circos genome plots (Figure 4).

Table 2. Tnt1 insertion distribution on the Medicago truncatula R108 Hi-C genome.

\begin{tabular}{ccc}
\hline Mapping Description & No of FSTs & \% of Total FSTs \\
\hline FSTs mapped to Chromosome 1 & 27,902 & 12.61 \\
FSTs mapped to Chromosome 2 & 24,559 & 11.1 \\
FSTs mapped to Chromosome 3 & 27,679 & 12.51 \\
FSTs mapped to Chromosome 4 & 26,975 & 12.19 \\
FSTs mapped to Chromosome 5 & 25,313 & 11.44 \\
FSTs mapped to Chromosome 6 & 16,433 & 7.43 \\
FSTs mapped to Chromosome 7 & 25,451 & 11.5 \\
FSTs mapped to Chromosome 8 & 27,115 & 12.25 \\
Total mapped to 8 chromosomes & 201,427 & 91.03 \\
Total mapped to non Chr & 1361 & 0.62 \\
scaffolds & & \\
\hline
\end{tabular}

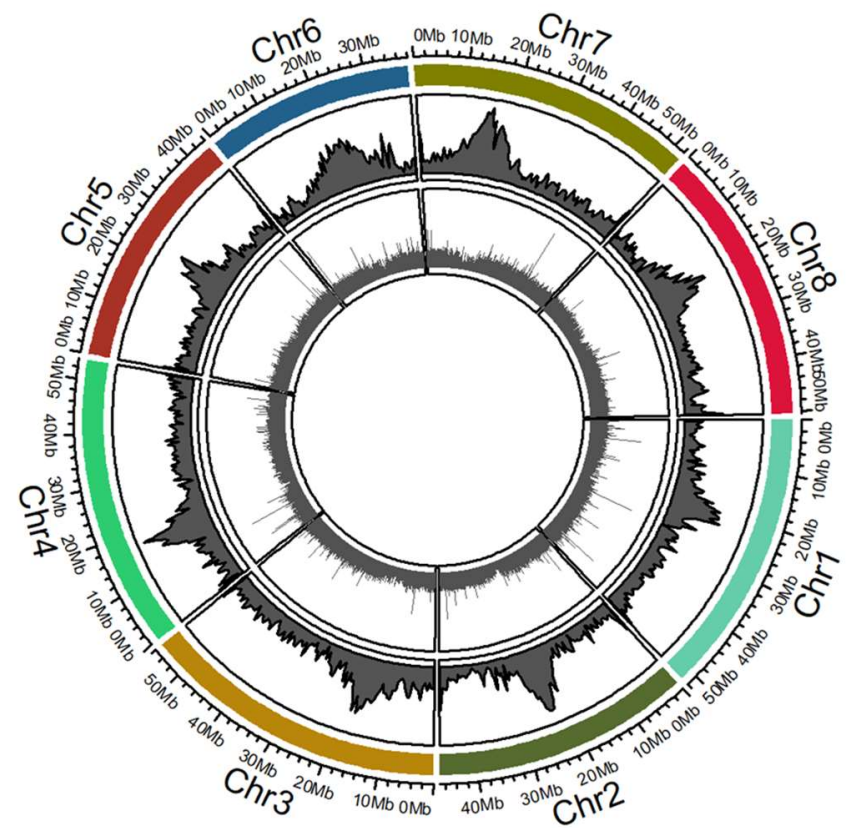

Figure 4. Circular genomic visualization of Tnt1 insertions in Medicago truncatula R108 genome. The figure was generated using the $\mathrm{R}$ statistical platform in the Rcircos package. The outer band (outer circle) has chromosome locations (Chr1-Chr8). Each of the chromosome regions was divided into $500 \mathrm{~Kb}$ bins and plotted as bins with specific genomic locations. The first band of the circle represents the GC percentage of the chromosome regions specific to those divided bins. The second inner circle represents Tnt1 insertions (as a measure of their FST lengths) in different chromosomes of the MedtrR108_hic assembly. 


\subsection{Comparison of Tnt1 Insertions Using M. truncatula R108 Hi-C or A17 v5.0 Genic Regions and Functional Annotation of Genes with Insertions}

In the M. truncatula A17 v5.0 reference genome [14], 44,624 genes were predicted and annotated. Our Hi-C assembly predicted 39,027 genes in M. truncatula R108. From 202,788 high-confidence FSTs (Table S18), there were 24,052 genes with exact Tnt 1 insertion sites $(61.62 \%)$ in the R108 Hi-C assembly (Tables S19-S20). We found a similar percentage of genes $(60 \% ; 26,717$ genes) in the $M$. truncatula A17 v5.0 reference genome with Tnt 1 insertion in at least one gene (Table S21). A list of the GO annotations analyzed for genes with Tnt1 insertions and the gene groups are summarized in Table S22. In the R108 HiC version, there were at least 19,008 genes (48.7\%) with more than one Tnt1 insertion, contrasting with 18,352 genes $(41.12 \%)$ in the M. truncatula A17 v5.0 reference genome (Tables S20-S21). There were at least 12,746 genes (32.65\%) with at least four Tnt1 insertions in the R108 Hi-C assembly, contrasting with $22.29 \%$ of the genes (9949 genes) in the $M$. truncatula A17 v5.0 reference genome (Tables S20-S21). An average of 4.07 Tnt1 insertions per gene was observed in the MedtrR108_hic assembly compared to 4.33 insertions per gene in M. truncatula A17 v5.0 (Tables S20-S21).

The most frequently hit gene when M. truncatula A17 v5.0 genome was used for analysis is MtrunA17Chr5g0441701 (putative peptidyl prolyl isomerase), with 135 Tnt1 insertions (Table S20), while the two genes with more Tnt1 insertions when MedtrR108_hic assembly was used for analysis are MedtrR108_hic. Hi-C_scaffold_8.3452 (Eukaryotic and viral aspartyl proteases active site protein) and MedtrR108_hic. Hi-C_scaffold_2.2064 (RHN73856.1 putative FAS1 domain-containing protein) with 143 and 139 Tnt1 insertions, respectively (Table S21). The genes that did not have insertions were also identified (Tables S20-S21). It is reasonable to assume that Tnt1 in the existing insertion population disrupts majority of genes in the M. truncatula genome. GO ontology and annotation were performed for all genes with frequent insertions and insertions into genes with less frequency (Table S22).

AgriGO v2.0 [28] analysis was used to enrich the frequently inserted 7737 genes in GO categories, which were selected based on genes that are inserted more than the average insertion number (i.e., 4.33 insertions per gene). The results showed that these frequently inserted genes fall into the following five pathways: stress, signaling, secondary metabolism, transport, and nucleotide metabolism (Table S22 and Figure S1A). The significant GO terms under the biological processes are response to stress, response to stimulus, defense response, protein phosphorylation, and transmembrane transport (Table S22). The significant GO terms under molecular functions are ATP binding, active transmembrane transporter activity, protein tyrosine kinase activity, and transporter activity (Figure S1B). The GO enrichment analysis revealed similar results to the pathway analysis and corresponded with the previously reported data [12].

\subsection{Tnt1 Insertions in Genes in the Syntenic Regions}

Syntenic regions between A17 v5.0 and R108 v1.0 genome were obtained from the publicly released v1.0 [14]. The syntenic region between A17 v5.0 and MedtrR108_hic syntenic genes could be arranged into a smaller number of larger blocks than the A17 Mt5.0 versus R108 v1.0 syntenic genes. A total of 25,548 syntenic genes were identified between A17 Mt5.0 and MedtrR108_hic (Table S12), which could be arranged in 59 collinear blocks. The largest block (no. 54) contained 2574 genes, while the smallest block (no. 49) contained four genes. We identified 17,766 genes present in all syntenic blocks combined between A17 and R108 (Table S23). Each of the Tnt1 genic insertions in the syntenic regions and the GO annotation is presented in Table S22. Individual gene numbers from each block are identified and presented as a supplemental table (Table S24). Six syntenic blocks $(54,25,9$, 36,12 , and 31) have more than 1000 genes with Tnt1 insertions (Table S24). The highest number of genic Tnt1 insertions are in Block 54 with 1787 genes (Tables S23-S24). 


\section{Discussion}

The MedtrR108_hic assembly is a significant improvement on the R108 v1.0 assembly, with its smaller number of larger scaffolds, higher scaffold N50 value and improved CEGMA results. While fewer genes were annotated in the Hi-C assembly, the gene content appeared to be more complete than the R108 v1.0 annotation, as reflected in the BLAST and BUSCO results for MedtrR108_hic [20] processed through the MAKER-P pipeline [29] for annotation; only ab initio gene predictions from RNA-Seq alignments were used as the source of evidence. In the current study, a combination of ab initio gene predictions from RNA-Seq alignments and evidence from protein homology studies were used for annotation via the BRAKER2 [30] pipeline and EvidenceModeler [31]. The latter tool primarily leverages the ab initio predictions as its source of gene model components, and then leverages the protein and transcript alignment data to guide its choice of best models. Therefore, any ab initio predictions not supported by the protein/transcript alignments are discarded. This strict filtering could explain why we observed a reduction in the number of genes. Additionally, the RNA-Seq libraries used to annotate the Hi-C assembly were derived from root tissue [20] and leaf tissue (data generated in-house). However, the R108 v1.0 assembly was annotated using RNA-Seq data from root tissue only [20]. This could explain why the current annotation is more complete.

The abnormal conformation of chromosomes 4 and 8 in genotype A17 is well-known $[14,27]$. The smaller number of larger collinear blocks identified between A17 Mt5.0 and MedtrR108_hic, coupled with the larger $17 \mathrm{Mb}$ translocation between A17 Mt5.0 chromosome 4 and MedtrR108_hic chromosome 8, reflects the more contiguous nature of the Hi-C assembly than R108 v1.0. Furthermore, the absence of the three additional breakpoints (BKPT 2, 3, and 4) identified by Pecrix et al. in the Hi-C assembly when comparing A17 Mt5.0 and R108 v1.0 suggests that these breakpoints occurred as a result of the more fragmented nature of the R108 v1.0 assembly or the presence of errors in the assembly. Therefore, it is unlikely that these breakpoints represent true structural variations in A17. On the other hand, the inversion in A17 Mt5.0 for both MedtrR108_hic and R108 v1.0 indicates that this structural variation is real and constitutes a second distinctive structural feature of the A17 genotype. This inversion was also visible when A17 Mt5.0 was compared with the genetic maps of Medicago sativa and Pisum sativa, the species most closely related to M. truncatula [14].

Tnt1 insertion lines have become more and more popular due to their powerful, versatile applications in forward and reverse genetics. The Tnt 1 lines were generated in the R108 background due to its high transformation and regeneration efficiency. The A17 and R108 genomes significantly differed due to their phylogenetic distance [32]. Though most genes in both genomes have high similarity, there are a significant number of genes that have moderate similarity, which will cause ambiguity in determining whether a BLAST search result of a gene with the A17 sequence is a true hit in the Tnt1 FST database. Therefore, a high-quality R108 genome assembly was needed. Compared to the genome R108 v1.0, the assembly quality of MedtrR108_hic has significantly improved, especially in the syntenic translocation regions, where Tnt1 FST mapping is more accurate in the MedtrR108_hic genome.

Genome-editing technology, especially Clustered Regularly Interspaced Short Palindromic Repeat/CRISPR-associated protein 9 (CRISPR/Cas9) technology, has become more powerful and applicable to many plant species, including M. truncatula. CRISPR/Cas9 is an innovative technology, offering excellent opportunities for plant genetics and functional genomics research. Its advantages include target specificity, effectiveness, precision, and feasibility for multiple genome manipulation options [33]. Accurate plant gene sequences are critical for gene editing. The improved genome editing efficiency in M. truncatula [34] should increase CRISPR/Cas9 technology use. Due to significant differences in the transformation efficiencies between A17 and R108, R108 is the first choice for genome editing practices. The improved genome assembly of R108 provides a solid foundation for future genome editing research in the legume community. 


\section{Materials and Methods}

\subsection{Hi-C Library Preparation and Sequencing}

In situ Hi-C was performed as described previously [18] using frozen leaves from Medicago truncatula cv. R108. Briefly, frozen leaf tissue was crosslinked, ground and then lysed with nuclei permeabilized but still intact. DNA was then restricted with $M b o \mathrm{I}$ restriction enzyme and the overhangs filled in incorporating a biotinylated base. Free ends were then ligated together in situ. Crosslinks were reversed, the DNA was sheared to 300-500 bp and then biotinylated ligation junctions were recovered with streptavidin beads.

Standard Illumina library construction protocol was used for DNA sequencing. Briefly, DNA was end-repaired using a combination of T4 DNA polymerase, Escherichia coli DNA Pol I large fragment (Klenow polymerase), and T4 polynucleotide kinase. The blunt, phosphorylated ends were treated with Klenow fragment ( $3^{\prime}$ to $5^{\prime}$ exo minus) and dATP to yield a protruding 3- 'A' base for ligation of Illumina's adapters which have a single ' $\mathrm{T}$ ' base overhang at the 3 ' end. After adapter ligation, DNA was PCR amplified with Illumina primers for 14 cycles and library fragments of 400-600 bp (insert plus adaptor and PCR primer sequences) were purified using SPRI beads. The purified DNA was captured on an Illumina flow cell for cluster generation. Libraries were sequenced on the NextSeq500 following the manufacturer's protocols. The same R108 lineage used for generating Tnt1 insertion lines was used for Hi-C. The resulting library was sequenced to yield approximately $48 \times$ coverage of the $M$. truncatula genome.

\subsection{Genome Assembly}

The Hi-C library was processed against the R108 v1.0 genome assembly [20] the Juicer pipeline [35]. The assembly was performed as described [19,21]. Briefly, after excluding scaffolds shorter than $1 \mathrm{~Kb}$, the 3D De Novo Assembly (3D-DNA) pipeline was run using the in situ Hi-C data to anchor, order, orient, and correct misjoins in the R108 v1.0 scaffolds. Lastly, a manual refinement step was performed using Juicebox Assembly Tools [21]. The resulting contact maps were visualized using the 3D-DNA and Juicebox visualization system $[19,21,36]$.

\subsection{Genome Annotations}

A multistep approach consisting of ab initio gene predictions, protein alignments, and transcript assembly was used to annotate the MedtrR108_hic reference genome assembly. RepeatModeler [37] v1.0.9. was used to identify interspersed repeats and low complexity DNA sequences in the assembly. These regions were soft-masked in the assembly using RepeatMasker [38] v4.0.8. The resulting soft-masked assembly was used as the input for BRAKER2 [30] for ab initio gene prediction using GeneMark-ET v4.33 [39] and AUGUSTUS v3.3.1 [40] based on alignments of RNA-Seq data. Prior to alignment, adapter sequences and low-quality bases were trimmed from the Illumina RNA-Seq libraries using Trimmomatic [41] v0.33. (sliding window, minimum quality score: 20). Trimmed libraries were aligned to MedtrR108_hic reference assembly using HISAT2 [42] v2.1.0. (insert size 0 to 1000). The resulting SAM files were converted to BAM format using SAMtools [43] v1.3, which were then merged prior to transcript assembly using Stringtie [44] v 1.3.5.

A total of $89,910 \mathrm{M}$. truncatula protein sequences were accessed and downloaded from NCBI (https:/ / www.ncbi.nlm.nih.gov/, accessed on 4 April 2019). These sequences were aligned to the soft-masked reference using Exonerate [45] v2.2.0 (-softmasktarget -model protein2genome -showvulgar no -showalignment no -showquerygff no-showtargetgff yes - percent 80).

Finally, EvidenceModeler [31] v1.1.1. was used to combine the gene predictions from GeneMark-ET and AUGUSTUS, protein alignments from Exonerate, and the assembled transcripts from Stringtie to obtain the final gene set. 


\subsection{Assessment of Genome Assembly and Annotation Quality}

Assessment of the Hi-C genome assembly quality and completeness was performed via CEGMA [22] v2.5. to identify the presence of CEGs. BUSCO [23] v4.14. was run using the eudicotyledons_odb10 dataset in protein mode to evaluate the annotation quality.

\subsection{Functional Annotation of the Predicted Genes}

A protein BLAST search (blastp) was performed by aligning the predicted proteins to several databases using BLAST [46] v2.2.29 (minimum e-value 1e-5). The databases used were the A17 Mt5.0 proteins [14], SwissProt [25], TrEMBL [37], TAIR10 (https:// www.arabidopsis.org/, accessed on 17 March 2021), RefSeq [26], and Phytozome [24] v13, accessed and downloaded on 30 November 2020. For each predicted protein, the hit with the highest score and lowest e-value was chosen as annotation. KEGG numbers were assigned to all predicted proteins using BLASTKOALA (taxonomy group: Plants, KEGG GENES database: family_eukaroytes) [47]. The predicted genes were annotated with GO terms and mined for protein domains using InterProScan [48] v5.45-80.0 and the following databases: TIGRFAM, ProDom, PANTHER, Pfam, PrositeProfiles, PrositePatterns, Coils, SUPERFAMILY, SFLD, SMART, PRINTS, MobiDBLite, and PIRSF.

\subsection{Genome Alignment and Detection of Chromosomal Translocation}

Whole genome alignments were performed between MedtrR108_hic and A17 Mt5.0 (GenBank Accession no. GCA_003473485.2) using minimap 2 [32] v2.17 (-x asm5). The alignments were filtered [14] (primary alignments only [tp:A:P]; alignment block length > $10 \mathrm{~Kb}$; approximate per-base sequence divergence (dv) score lower than 0.8$)$, and visualized using D-genies [49] v1.2.0 (dot plot) and Circos [50] v0.69 (circular genome plot).

The python version of MCScan [51] was used to identify syntenic regions and their corresponding genes between MedtrR108_hic and A17 Mt.5.0, and R108 v1.0 (GenBank Accession no. GCA_002024945.1) and A17 Mt5.0. A syntenic region was defined as one with at least 10 shared genes $(-(-\operatorname{minspan}=10)$.

\subsection{Mapping of Tnt1 Insertion Lines and Functional Gene Group Analysis}

To accurately identify Tnt1 insertion sites in the M. truncatula genome, all FST sequences shorter than $50 \mathrm{bp}$, without the Tnt1 signature sequence ('CCCAACA,' 'CATCATCA' or 'TGATGATGTCC'), or the Tnt1 signature sequence outside the $28 \mathrm{bp}$ from the beginning or end of the FST sequence were discarded. The preprocessed reliable FST sequences were aligned to the M. truncatula A17 version4 (Mt4.0) or version5 (Mt5.0) and R108 Hi-C assembly reference genomes using BLASTN with an e-value threshold $\leq 1.00 \times 10^{-5}$. The FST sequences with best hit from BLAST analysis were further processed for downstream analysis. Only hits with at least $90 \%$ sequence identity were considered and used for functional gene group analysis. Functional gene group analysis was performed as described elsewhere [12].

\section{Conclusions}

Using in situ Hi-C data, we improved the M. truncatula cv. R108 genome assembly by correcting misjoins and ordering and orienting scaffolds to generate eight chromosomelength large scaffolds that correspond to the eight chromosomes in the A17 reference genome. Compared to the previous version (v1.0) of the R108 genome, the newly assembled MedtrR108_hic genome is a significant improvement due to its smaller number of larger scaffolds, higher scaffold N50 value, and improved CEGMA results. MedtrR108_hic also provides insight into how to accurately predict syntenies in the chromosome 4/8 translocation regions between A17 and R108. Furthermore, mapping the Tnt1 insertion landscape onto the current reference assembly provides a much-needed foundational resource for functional genomics studies in the legume community. 


\section{Patents}

O.D., M.P., C.L, and E.L.A. are inventors on U.S. provisional patent application $62 / 347,605$ filed 8 June 2016, by the Baylor College of Medicine and the Broad Institute, relating to the assembly methods in this manuscript.

Supplementary Materials: The following are available online at https:/ / www.mdpi.com/article/10 .3390/ijms22094326/s1, Additional supporting information (supporting Figure S1, Pathway analysis and GO annotation of genes with Tnt1 insertions, and Tables S1-S22) are provided as separate files.

Author Contributions: Conceptualization, P.K. and K.SM.; methodology, C.L., P.K. and M.P.; formal analysis, O.D., B.H., R.S.N., M.P., C.L., K.SM., J.W. and E.L.A.; resources, P.K. and K.SM. and E.L.A.; writing—original draft preparation, P.K., R.S.N., B.H., J.W. and K.SM.; writing—review and editing, P.K., O.D., R.S.N., B.H., J.W. and K.SM. All authors have read and agreed to the published version of the manuscript.

Funding: This work was supported by NSF Physics Frontiers Center Award (PHY-1427654, Center for Theoretical Biological Physics), the Welch Foundation (Q-1866), Noble Research Institute, LLC. McNair Medical Institute Scholar Award, NIH 4D Nucleome Grant (U01HL130010) and NIH Encyclopedia of DNA Elements (ENCODE) Mapping Center Award (UM1HG009375) to E.L.A., and NSF Plant Genome Grant (IOS-1733470) to K.SM.

Institutional Review Board Statement: Not applicable.

Informed Consent Statement: Not applicable.

Data Availability Statement: All the data is available under the DNA Zoo BioProject accession PRJNA512907.

Acknowledgments: We gratefully acknowledge the frozen leaf sample provided by Scott Schaeffer at the Nakata Lab, Baylor College of Medicine. P.K. acknowledges The University of Western Australia, with additional computational resources and support from the Pawsey Supercomputing Centre, and funding from the Australian Government and Government of Western Australia.

Conflicts of Interest: The authors declare no conflict of interest.

\section{References}

1. Tilman, D.; Balzer, C.; Hill, J.; Befort, B.L. Global food demand and the sustainable intensification of agriculture. Proc. Natl. Acad. Sci USA 2011, 108, 20260-20264. [CrossRef] [PubMed]

2. Chikowo, R.; Mapfumo, P.; Nyamugafata, P.; Giller, K.E. Maize productivity and mineral N dynamics following different soil fertility management practices on a depleted sandy soil in Zimbabwe. Agric. Ecosyst. Environ. 2004, 102, 119-131. [CrossRef]

3. Saha, N.; Mandal, B. Soil health-A precondition for crop production. In Microbial STRATEgies for Crop Improvement; Springer: Cham, Switzerland, 2009; pp. 161-184.

4. Tang, H.B.; Krishnakumar, V.; Bidwell, S.; Rosen, B.; Chan, A.N.; Zhou, S.G.; Gentzbittel, L.; Childs, K.L.; Yandell, M.; Gundlach, H.; et al. An improved genome release (version Mt4.0) for the model legume Medicago truncatula. BMC Genom. 2014, 15. [CrossRef]

5. Michno, J.M.; Wang, X.B.; Liu, J.Q.; Curtin, S.J.; Kono, T.J.Y.; Stupar, R.M. CRISPR/Cas mutagenesis of soybean and Medicago truncatula using a new web-tool and a modified Cas9 enzyme. Genet. Modif. Crop Food 2015, 6, 243-252. [CrossRef]

6. Cermak, T.; Curtin, S.J.; Gil-Humanes, J.; Cegan, R.; Kono, T.J.Y.; Konecna, E.; Belanto, J.J.; Starker, C.G.; Mathre, J.W.; Greenstein, R.L.; et al. a multipurpose toolkit to enable advanced genome engineering in plants. Plant Cell 2017, 29, 1196-1217. [CrossRef]

7. Curtin, S.J.; Xiong, Y.; Michno, J.M.; Campbell, B.W.; Stec, A.O.; Cermak, T.; Starker, C.; Voytas, D.F.; Eamens, A.L.; Stupar, R.M. CRISPR/Cas9 and TALENs generate heritable mutations for genes involved in small RNA processing of Glycine max and Medicago truncatula. Plant Biotechnol. J. 2018, 16, 1125-1137. [CrossRef] [PubMed]

8. Meng, Y.Y.; Hou, Y.L.; Wang, H.; Ji, R.H.; Liu, B.; Wen, J.Q.; Niu, L.F.; Lin, H. Targeted mutagenesis by CRISPR/Cas9 system in the model legume Medicago truncatula. Plant Cell Rep. 2017, 36, 371-374. [CrossRef] [PubMed]

9. Gao, F.; Wang, X.; Li, X.M.; Xu, M.Y.; Li, H.Y.; Abla, M.; Sun, H.G.; Wei, S.J.; Feng, J.C.; Zhou, Y.J. Long-read sequencing and de novo genome assembly of Ammopiptanthus nanus, a desert shrub. Gigascience 2018, 7. [CrossRef]

10. Tadege, M.; Wen, J.Q.; He, J.; Tu, H.D.; Kwak, Y.; Eschstruth, A.; Cayrel, A.; Endre, G.; Zhao, P.X.; Chabaud, M.; et al. Large-scale insertional mutagenesis using the Tnt1 retrotransposon in the model legume Medicago truncatula. Plant J. 2008, 54, 335-347. [CrossRef]

11. Young, N.D.; Debelle, F.; Oldroyd, G.E.D.; Geurts, R.; Cannon, S.B.; Udvardi, M.K.; Benedito, V.A.; Mayer, K.F.X.; Gouzy, J.; Schoof, H.; et al. The Medicago genome provides insight into the evolution of rhizobial symbioses. Nature 2011, 480, 520-524. [CrossRef] [PubMed] 
12. Sun, L.; Gill, U.S.; Nandety, R.S.; Kwon, S.; Mehta, P.; Dickstein, R.; Udvardi, M.K.; Mysore, K.S.; Wen, J.Q. Genome-wide analysis of flanking sequences reveals that Tnt1 insertion is positively correlated with gene methylation in Medicago truncatula. Plant J. 2019, 98, 1106-1119. [CrossRef] [PubMed]

13. Blanc, G.; Wolfe, K.H. Widespread paleopolyploidy in model plant species inferred from age distributions of duplicate genes. Plant Cell 2004, 16, 1667-1678. [CrossRef] [PubMed]

14. Pecrix, Y.; Staton, S.E.; Sallet, E.; Lelandais-Brere, C.; Moreau, S.; Carrere, S.; Blein, T.; Jardinaud, M.F.; Latrasse, D.; Zouine, M.; et al. Whole-genome landscape of Medicago truncatula symbiotic genes. Nat. Plants 2018, 4, 1017-1025. [CrossRef] [PubMed]

15. Stevens, C.; Smith, L.; La Thangue, N.B. Chk2 activates E2F-1 in response to DNA damage. Nat. Cell Biol. 2003, 5, 401-409. [CrossRef]

16. Lieberman-Aiden, E.; van Berkum, N.L.; Williams, L.; Imakaev, M.; Ragoczy, T.; Telling, A.; Amit, I.; Lajoie, B.R.; Sabo, P.J.; Dorschner, M.O.; et al. Comprehensive mapping of long-range interactions reveals folding principles of the human genome. Science 2009, 326, 289-293. [CrossRef]

17. Burton, J.N.; Adey, A.; Patwardhan, R.P.; Qiu, R.L.; Kitzman, J.O.; Shendure, J. Chromosome-scale scaffolding of de novo genome assemblies based on chromatin interactions. Nat. Biotechnol. 2013, 31, 1119-1125. [CrossRef]

18. Rao, S.S.P.; Huntley, M.H.; Durand, N.C.; Stamenova, E.K.; Bochkov, I.D.; Robinson, J.T.; Sanborn, A.L.; Machol, I.; Omer, A.D.; Lander, E.S.; et al. A 3D map of the human genome at kilobase resolution reveals principles of chromatin looping. Cell 2014, 159, 1665-1680. [CrossRef]

19. Dudchenko, O.; Batra, S.S.; Omer, A.D.; Nyquist, S.K.; Hoeger, M.; Durand, N.C.; Shamim, M.S.; Machol, I.; Lander, E.S.; Aiden, A.P.; et al. De novo assembly of the Aedes aegypti genome using Hi-C yields chromosome-length scaffolds. Science 2017, 356, 92-95. [CrossRef]

20. Moll, K.M.; Zhou, P.; Ramaraj, T.; Fajardo, D.; Devitt, N.P.; Sadowsky, M.J.; Stupar, R.M.; Tiffin, P.; Miller, J.R.; Young, N.D.; et al. Strategies for optimizing BioNano and Dovetail explored through a second reference quality assembly for the legume model, Medicago truncatula. BMC Genom. 2017, 18. [CrossRef]

21. Dudchenko, O.; Shamim, M.S.; Batra, S.S.; Durand, N.C.; Musial, N.T.; Mostofa, R.; Pham, M.; St Hilaire, B.G.; Yao, W.; Stamenova, E. The juicebox assembly tools module facilitates de novo assembly of mammalian genomes with chromosome-length scaffolds for under \$1000. Biorxiv 2018, 254797.

22. Parra, G.; Bradnam, K.; Korf, I. CEGMA: A pipeline to accurately annotate core genes in eukaryotic genomes. Bioinform. 2007, 23, 1061-1067. [CrossRef]

23. Simao, F.A.; Waterhouse, R.M.; Ioannidis, P.; Kriventseva, E.V.; Zdobnov, E.M. BUSCO: Assessing genome assembly and annotation completeness with single-copy orthologs. Bioinformatics 2015, 31, 3210-3212. [CrossRef] [PubMed]

24. Goodstein, D.M.; Shu, S.Q.; Howson, R.; Neupane, R.; Hayes, R.D.; Fazo, J.; Mitros, T.; Dirks, W.; Hellsten, U.; Putnam, N.; et al. Phytozome: A comparative platform for green plant genomics. Nucleic Acids Res. 2012, 40, D1178-D1186. [CrossRef] [PubMed]

25. Boeckmann, B.; Bairoch, A.; Apweiler, R.; Blatter, M.C.; Estreicher, A.; Gasteiger, E.; Martin, M.J.; Michoud, K.; O’Donovan, C.; Phan, I.; et al. The SWISS-PROT protein knowledgebase and its supplement TrEMBL in 2003. Nucleic Acids Res. 2003, 31, 365-370. [CrossRef] [PubMed]

26. Pruitt, K.D.; Tatusova, T.; Maglott, D.R. NCBI reference sequences (RefSeq): A curated non-redundant sequence database of genomes, transcripts and proteins. Nucleic Acids Res. 2007, 35, D61-D65. [CrossRef]

27. Kamphuis, L.G.; Williams, A.H.; D'Souza, N.K.; Pfaff, T.; Ellwood, S.R.; Groves, E.J.; Singh, K.B.; Oliver, R.P.; Lichtenzveig, J. The Medicago truncatula reference accession A17 has an aberrant chromosomal configuration. New Phytol. 2007, 174, 299-303. [CrossRef] [PubMed]

28. Tian, T.; Liu, Y.; Yan, H.Y.; You, Q.; Yi, X.; Du, Z.; Xu, W.Y.; Su, Z. agriGO v2.0: A GO analysis toolkit for the agricultural community, 2017 update. Nucleic Acids Res. 2017, 45, W122-W129. [CrossRef]

29. Campbell, M.S.; Law, M.Y.; Holt, C.; Stein, J.C.; Moghe, G.D.; Hufnagel, D.E.; Lei, J.K.; Achawanantakun, R.; Jiao, D.; Lawrence, C.J.; et al. MAKER-P: A tool kit for the rapid creation, management, and quality control of plant genome annotations. Plant Physiol. 2014, 164, 513-524. [CrossRef]

30. Hoff, K.J.; Lomsadze, A.; Borodovsky, M.; Stanke, M. Whole-Genome Annotation with BRAKER. Methods Mol. Biol. 2019, 1962, 65-95.

31. Haas, B.J.; Salzberg, S.L.; Zhu, W.; Pertea, M.; Allen, J.E.; Orvis, J.; White, O.; Buell, C.R.; Wortman, J.R. Automated eukaryotic gene structure annotation using EVidenceModeler and the program to assemble spliced alignments. Genome Biol. $2008,9$. [CrossRef]

32. Li, H. Minimap2: Pairwise alignment for nucleotide sequences. Bioinformatics 2018, 34, 3094-3100. [CrossRef] [PubMed]

33. Voytas, D.F.; Gao, C.X. Precision genome engineering and agriculture: Opportunities and regulatory challenges. PLoS Biol. 2014, 12. [CrossRef] [PubMed]

34. Wolabu, T.W.; Park, J.-J.; Chen, M.; Cong, L.; Ge, Y.; Jiang, Q.; Debnath, S.; Li, G.; Wen, J.; Wang, Z. Improving the genome editing efficiency of CRISPR/Cas9 in Arabidopsis and Medicago truncatula. Planta 2020, 252, 1-14. [CrossRef]

35. Durand, N.C.; Robinson, J.T.; Shamim, M.S.; Machol, I.; Mesirov, J.P.; Lander, E.S.; Aiden, E.L. juicebox provides a visualization system for hi-c contact maps with unlimited zoom. Cell Syst. 2016, 3, 99-101. [CrossRef]

36. Durand, N.C.; Shamim, M.S.; Machol, I.; Rao, S.S.P.; Huntley, M.H.; Lander, E.S.; Aiden, E.L. Juicer provides a one-click system for analyzing loop-resolution hi-c experiments. Cell Syst. 2016, 3, 95-98. [CrossRef] [PubMed] 
37. Robert Hubley, A.S. RepeatModeler Open-1.0, Version 1.0. 2008.

38. Robert Hubley, A.S. RepeatMasker Open-4.0, Version 4.0, 2013-2015.

39. Lukashin, A.V.; Borodovsky, M. GeneMark.hmm: New solutions for gene finding. Nucleic Acids Res. 1998, 26, 1107-1115. [CrossRef] [PubMed]

40. Stanke, M.; Waack, S. Gene prediction with a hidden Markov model and a new intron submodel. Bioinformatics 2003, 19, Ii215-Ii225. [CrossRef]

41. Bolger, A.M.; Lohse, M.; Usadel, B. Trimmomatic: A flexible trimmer for Illumina sequence data. Bioinformatics 2014, 30, 2114-2120. [CrossRef]

42. Kim, D.; Landmead, B.; Salzberg, S.L. HISAT: A fast spliced aligner with low memory requirements. Nat. Methods 2015, 12, 357-360. [CrossRef]

43. Li, H.; Handsaker, B.; Wysoker, A.; Fennell, T.; Ruan, J.; Homer, N.; Marth, G.; Abecasis, G.; Durbin, R.; Proc, G.P.D. The Sequence Alignment/Map format and SAMtools. Bioinformatics 2009, 25, 2078-2079. [CrossRef]

44. Pertea, M.; Pertea, G.M.; Antonescu, C.M.; Chang, T.C.; Mendell, J.T.; Salzberg, S.L. StringTie enables improved reconstruction of a transcriptome from RNA-seq reads. Nat. Biotechnol. 2015, 33, 290-295. [CrossRef] [PubMed]

45. Slater, G.S.; Birney, E. Automated generation of heuristics for biological sequence comparison. BMC Bioinform. 2005, 6. [CrossRef] [PubMed]

46. Johnson, M.; Zaretskaya, I.; Raytselis, Y.; Merezhuk, Y.; McGinnis, S.; Madden, T.L. NCBI BLAST: A better web interface. Nucleic Acids Res. 2008, 36, D543-D546. [CrossRef] [PubMed]

47. Kanehisa, M.; Sato, Y.; Morishima, K. BlastKOALA and GhostKOALA: KEGG Tools for functional characterization of genome and metagenome sequences. J. Mol. Biol. 2016, 428, 726-731. [CrossRef]

48. Jones, P.; Binns, D.; Chang, H.Y.; Fraser, M.; Li, W.Z.; McAnulla, C.; McWilliam, H.; Maslen, J.; Mitchell, A.; Nuka, G.; et al. InterProScan 5: Genome-scale protein function classification. Bioinformatics 2014, 30, 1236-1240. [CrossRef]

49. Cabanettes, F.; Klopp, C. D-GENIES: Dot plot large genomes in an interactive, efficient and simple way. PeerJ 2018, 6, e4958. [CrossRef] [PubMed]

50. Krzywinski, M.; Schein, J.; Birol, I.; Connors, J.; Gascoyne, R.; Horsman, D.; Jones, S.J.; Marra, M.A. Circos: An information aesthetic for comparative genomics. Genome Res. 2009, 19, 1639-1645. [CrossRef] [PubMed]

51. Tang, H.B.; Bowers, J.E.; Wang, X.Y.; Ming, R.; Alam, M.; Paterson, A.H. Perspective-Synteny and collinearity in plant genomes. Science 2008, 320, 486-488. [CrossRef] 